\title{
On Reproducing Kernels and Quantization of States
}

\author{
Anatol Odzijewicz \\ Institute of Physics, Warsaw University Division in Białystok, 15-424 Białystok, PL-41 Lipowa, \\ Poland
}

\begin{abstract}
Quantization of a mechanical system with the phase space a Kähler manifold is studied. It is shown that the calculation of the Feynman path integral for such a system is equivalent to finding the reproducing kernel function. The proposed approach is applied to a scalar massive conformal particle interacting with an external field which is described by deformation of a Hermitian line bundle structure.
\end{abstract}

\section{Introduction}

In the case of ordinary quantum mechanics the space of pure states is a projective complex Hilbert space. As a consequence the role of complex numbers is crucial in the description of quantum phenomena. On the other hand, the classical mechanical systems are described in terms of real differential geometry. However, many leading quantized classical systems have complex differential manifolds as phase spaces. Let us give a few examples: 1 ) the space of orbits of the $n$-dimensional harmonic isotropic oscillator is $\mathbb{C P}(n-1)$ (see [6]);2) the phase space of a spin system is given by $\mathbb{C P}(1)$; 3) $\mathbb{C P}(1) \times \mathbb{C P}(1)$ is the phase space of orbits corresponding to the negative energy level in the Kepler problem (see [16]). The twistor theory provides us also with a wide class of complex phase spaces. In general they are realized as the orbits of the conformal group on twistor flag spaces (see [10]). In particular, the space of positive projective twistors is the phase of the photon with positive helicity (see $[13,14]$ ). Finally one should emphasize the important role of the Bargmann-Fock-Segal representation in quantum mechanics (because of its holomorphicity).

In Sect. 2 of this paper we study the quantization of a classical mechanical system where the phase space $M$ is a Kähler manifold. The basic feature which distinguishes such a system among the others is the possibility of quantization of classical states. This means that in some special case, when the Hilbert space of quantum states satisfies some condition of ampleness (see Propositions 2 and 3), one can embed $M$ into $\mathbb{C P}(\mathscr{M})$, where $\mathscr{M}$ consists of square integrable holomorphic sections of a Hermitian line bundle $\mathbb{E}$ over $M$. Using this embedding one 
can calculate the transition probability amplitude from one point of $M$ to another. In such a way, we find the interpretation of the normalized reproducing kernel function as the transition probability amplitude between any two points of complex phase space $M$ (see Sect. 2). The above interpretation is possible if and only if the holomorphic and metric structures of $\mathbb{E}$ satisfy a certain geometrical condition, which in coordinate terms becomes the complex Monge-Ampère equation. The rules of superposition and multiplication of transition probability amplitudes allow us to calculate them along any path. On the other hand, summing up contributions from all paths we obtain the transition probability amplitude between two corresponding points of phase space in terms of the Feynman path integral.

In Sect. 3 we link this fact with the idea of Penrose that interaction can be described as a deformation of a complex structure. Therefore, the holomorphic structure of $\mathbb{E}$ plays the role of an external field. The path integral description provides us with a Lagrangian depending on this deformation.

The last section of this paper contains the application of the developed formalism to the case of a conformal scalar massive particle. The phase of a conformal scalar massive particle is taken to be an open subset $\mathbb{M}^{++} \subset G(2, \mathbb{T})$ of two-dimensional positive definite subspaces in twistor space $\mathbb{T}$ (see [11]). In the free case, i.e. when the holomorphic structure is not deformed we take as the quantum bundle $\mathbb{E}$ the tensor product of the tautological Hermitian bundle over $\mathrm{M}^{++}$. The above theory contains a parameter which has a natural interpretation as the Planck constant $h$. After introducing the external field, i.e. deforming the holomorphic and metric structures of $\mathbb{E}$, we obtain the model of a charged conformal scalar massive particle interacting with this field. Expanding this model in powers of $h$ we find that the linear approximation of it becomes a model of a charged scalar massive particle in the electromagnetic field.

Finally, let us emphasize that the purpose of this work is to indicate some relations between quantum mechanics and complex analysis. One of the most interesting results of this paper seems to be the reduction of path integration to the calculation of the reproducing kernel function for the Hilbert space of states [see (2.27) and (2.28)]. Although the explicit form of reproducing kernels is known for some special cases only (see e.g. [5]) we believe that this approach to quantum mechanical problems could be physically fruitful, see e.g. the formula (4.23). We would also like to underline that complex analysis and complex differential geometry have proved to be useful in solving many other problems in theoretical physics, see e.g. $[1,14,15]$.

\section{The Quantization of Classical States}

In addition to quantizing observables we also want to quantize states of the classical physical system. By quantization of classical states we will understand an embedding $\mathscr{K}: M \rightarrow \mathbb{P C}(\mathscr{M})$ of classical phase space $M$ into quantum phase space $\mathbb{P C}(\mathscr{M})$ which is a complex projective Hilbert space (finite or infinite dimensional depending on the considered case). Because $\mathbb{P C}(\mathscr{M})$ has the canonical Kähler structure given by the Fubini-Study metric, we assume for consistency reasons that $M$ is a Kähler manifold, too, and its symplectic structure $\omega$ is given by the Kähler form. 
This section consists of two parts. In the first one we discuss some necessary facts concerning geometric quantization in the Kählerian case. Moreover, we find the explicit form for the Kostant-Souriau quantization prescription. In the second part we expose the role of the reproducing kernel function in ordinary quantum mechanics.

Let $\mathbb{E} \stackrel{\pi}{\longrightarrow} M$ be a differentiable complex line bundle on an $n$-dimensional complex manifold $M$. Let us also assume that $\mathbb{E}$ admits the structure of a holomorphic vector bundle with trivializations $\Phi_{\alpha}: \pi^{-1}\left(\Omega_{\alpha}\right) \rightarrow \Omega_{\alpha} \times \mathbb{C}$, where $\left(\Omega_{\alpha} ; z_{\alpha}^{1}, \ldots, z_{\alpha}^{n}\right)$ is a holomorphic atlas on $M$. Fixing a metric structure $H$ on $\mathbb{E}$ one obtains the metric connection

$$
\nabla: C^{\infty}(M, \mathbb{E}) \rightarrow C^{\infty}\left(M, \mathbb{E} \otimes T^{*} M\right),
$$

(i.e. the connection consistent with holomorphic and metric structures of $\mathbb{E}$ ) on $\mathbb{E}$, see [4]. Assuming that the curvature $(1,1)$-form curv $\nabla$ is nonsingular, one can consider $M$ as a symplectic manifold with symplectic structure given by the form $\omega=i \operatorname{curv} \nabla$. It follows that $\mathbb{E}$ is a quantum bundle (see $[6,9,18]$ ), for which the connection and the metric structure are given by $\nabla$ and $H$ respectively. The complex structure defines the canonical Kähler polarization $F:=T^{(0,1)} M$. (Let us recall that the $n$-dimensional complex involutive distribution $F \subset T^{\mathbb{C}} M$ is called a polarization iff: distributions $F \cap \bar{F}$ and $F+\bar{F}$ have constant dimension and $F \cap \bar{F}$ is involutive, where $\bar{F}$ is the complex conjugation of $F$. If $F \cap \bar{F}=\{0\}$, then $F$ is called Kähler polarization.) Hence, the space of $F$-stable sections

$$
\Gamma_{F}(\mathbb{E}):=\left\{s \in \Gamma(M, \mathbb{E}): \nabla_{\varphi} s=0, \underset{\varphi \in \Gamma(F)}{\forall}\right\}
$$

is given as the vector space of holomorphic global sections $H^{0}(M, \mathcal{O}(\mathbb{E}))$.

By the definition, the complex Hilbert space of quantum states $\mathscr{M}$ will consist of holomorphic sections $s \in H^{0}\left(M, \mathcal{O}\left(\mathbb{E} \otimes T^{*(n, 0)} M\right)\right)$ which satisfy $\langle s, s\rangle<+\infty$, where the scalar product $\langle\cdot, \cdot\rangle$ is given by

$$
\langle s, t\rangle:=i^{n^{2}} \int_{M} H(s, t),
$$

with $s, t \in \mathscr{M}$. By definition $s$ and $t$ are $\mathbb{E}$-valued holomorphic $n$-forms, thus, $H(s, t)$ is an $(n, n)$-form. Therefore, because $M$ is an oriented manifold, the integral (2.1) is well defined. The proof of completeness of $\mathscr{M}$ can be found for example in [3]. In geometric quantization $\mathscr{M}$ is usually defined as a subspace of square integrable with respect to the Liouville measure - holomorphic sections of $\mathbb{E}$. However the above definition of $\mathscr{M}$ is more natural from the reproducing kernels theory point of view.

In order to eliminate those cases when $\mathscr{M}$ is not sufficiently ample, let us postulate the following condition:

$$
\underset{z^{1}, z^{2} \in M}{\forall} \underset{s_{1}, s_{2} \in \mathcal{M}}{\exists} \text { such that } \operatorname{det}\left[\begin{array}{cc}
\psi_{1 \alpha}\left(z^{1}\right), & \psi_{1 \beta}\left(z^{2}\right) \\
\psi_{2 \alpha}\left(z^{1}\right), & \psi_{2 \beta}\left(z^{2}\right)
\end{array}\right] \neq 0,
$$

where $s_{i}=\psi_{i \alpha} s_{\alpha} \otimes d z_{\alpha}^{1} \wedge \ldots \wedge d z_{\alpha}^{n}, \quad s_{i}=\psi_{i \beta} s_{\beta} \otimes d z_{\beta}^{1} \wedge \ldots \wedge d z_{\beta}^{n}, \quad i=1,2$, and $z^{1} \in \Omega_{\alpha}$ $z^{2} \in \Omega_{\beta}$. Here $s_{\alpha}: \Omega_{\alpha} \rightarrow \mathbb{E}$ and $d z_{\alpha}^{1} \wedge \ldots \wedge d z_{\alpha}^{n}$ are holomorphic frames defined by $s_{\alpha}:=\Phi_{\alpha}^{-1}\left(\Omega_{\alpha} \times\{1\}\right)$ and by holomorphic coordinates $\left(\Omega_{\alpha} ; z_{\alpha}^{1}, \ldots, z_{\alpha}^{n}\right)$ respectively. 

Let $g_{\alpha \gamma}: \Omega_{\alpha} \cap \Omega_{\gamma} \rightarrow \mathcal{O}^{*}$ be the cocycle defined by $s_{\alpha}=g_{\alpha \gamma} S_{\gamma}$, and let $\frac{\partial z_{\alpha}}{\partial z_{\gamma}}: \Omega_{\alpha} \cap \Omega_{\gamma} \rightarrow \mathcal{O}^{*}$ be
the cocycle defined by

$$
d z_{\alpha}^{1} \wedge \ldots \wedge d z_{\alpha}^{n}=\frac{\partial z_{\alpha}}{\partial z_{\gamma}} d z_{\gamma}^{1} \wedge \ldots \wedge d z_{\gamma}^{n}
$$

Then, for $z^{1} \in \Omega_{\alpha} \cap \Omega_{\gamma}$ and $z^{2} \in \Omega_{\beta} \cap \Omega_{\delta}$ we have

$$
\operatorname{det}\left[\begin{array}{cc}
\psi_{1 \alpha}\left(z^{1}\right), & \psi_{1 \beta}\left(z^{2}\right) \\
\psi_{2 \alpha}\left(z^{1}\right), & \psi_{2 \beta}\left(z^{2}\right)
\end{array}\right]=g_{\gamma \alpha}\left(z^{1}\right) g_{\delta \beta}\left(z^{2}\right) \frac{\partial z_{\gamma}}{\partial z_{\alpha}}\left(z^{1}\right) \frac{\partial z_{\delta}}{\partial z_{\beta}}\left(z^{2}\right) \operatorname{det}\left[\begin{array}{ll}
\psi_{1 \gamma}\left(z^{1}\right), & \psi_{1 \delta}\left(z^{2}\right) \\
\psi_{2 \gamma}\left(z^{1}\right), & \psi_{2 \delta}\left(z^{2}\right)
\end{array}\right]
$$

which shows that the condition (2.2) is independent on the trivialization of the bundle $\mathbb{E} \otimes T^{*(n, 0)} M$.

The equality: $\omega\lrcorner \gamma(f)=d f$ defines a homomorphism $\gamma:\left(C^{\infty}(M, \mathbb{R}),\{\cdot, \cdot\}\right)$ $\rightarrow(H(M),[\cdot, \cdot])$ of the Poisson Lie algebra $C^{\infty}(M, \mathbb{R})$ into Lie algebra of Hamiltonian vector fields on $M$. The Lie algebra of quantizable observables is defined as

$$
C_{F F}:=\left\{f \in C^{\infty}(M, \mathbb{R}):[\gamma(f), F] \subset F\right\} .
$$

The Kostant-Souriau quantization is given accordingly by

$$
C_{F F} \ni f \rightarrow \hat{f}:=\frac{1}{i}\left[\left(\nabla_{\gamma(f)}+i f\right) \otimes \mathscr{L}_{\gamma(f)}\right]
$$

where the Lie derivative $\mathscr{L}_{X}$ acts on the section, of the canonical bundle $T^{*(n, 0)} M$. It is merely a technical problem to show that $\hat{f}$ preserves $\Gamma_{F}(\mathbb{E})$ and $i\{\widehat{f, g}\}=[\hat{f}, \hat{g}]$ for $f, g \in C_{F F}$. For the detailed description of the geometric quantization procedure, see for example $[3,6,9]$.

Dealing with the Kählerian case we are able to describe the space of quantizable quantities explicitly. Namely, let us fix $\left[g_{\alpha \beta}\right] \in H^{1}\left(M, \mathcal{O}^{*}\right)$, where $g_{\alpha \beta}$ is the cocycle providing the holomorphic structure on $\mathbb{E}$. Let $\varphi$ be a holomorphic vector field on $M$. Acting by $\varphi$ on $\log g_{\alpha \beta}$ we remove the ambiguity due to the choice of the logarithm branch. Thus, $\left[\varphi\left(\log g_{\alpha \beta}\right)\right]$ depends only on $\left[g_{\alpha \beta}\right]$, and we obtain the map $\tau: H^{0}\left(M, \mathcal{O}\left(T^{(1,0)} M\right)\right) \rightarrow H^{1}(M, \mathcal{O})$, where $\tau(\varphi):=\left[\varphi\left(\log g_{\alpha \beta}\right)\right]$. Let $H_{F F}$ consist of Hamiltonian vector fields of the form $\varphi+\bar{\varphi}$, where $\varphi \in \operatorname{Ker} \tau$. Then we have:

Proposition 1. The following sequence of Lie algebras

is exact.

$$
0 \rightarrow \mathbb{R} \stackrel{\imath}{\longrightarrow} C_{F F} \stackrel{\gamma}{\longrightarrow} H_{F F} \rightarrow 0
$$

Proof. Since $0 \rightarrow \mathbb{R} \stackrel{\iota}{\longrightarrow} C^{\infty}(M) \stackrel{\gamma}{\longrightarrow} H(M) \rightarrow 0$ is an exact sequence and $C_{F F} \subset C^{\infty}(M)$ and $H_{F F} \subset H(M)$ are subalgebras, it is enough to show that $\gamma\left(C_{F F}\right)=H_{F F}$.

From $[\gamma(f), F] \subset F$ and $f=\bar{f}$ we obtain $\gamma(f)=\varphi+\bar{\varphi}$, where $\varphi \in H^{0}\left(M, \mathcal{O}\left(T^{(1,0)} M\right)\right)$. Because $\omega=i \bar{\partial} \partial \log \varrho_{\bar{\alpha} \alpha}$, this gives

$$
i \bar{\partial} \varphi\left(\log \varrho_{\bar{\alpha} \alpha}\right)-i \partial \bar{\varphi}\left(\log \varrho_{\bar{\alpha} \alpha}\right)=\partial f+\bar{\partial} f
$$

on $\Omega_{\alpha}$, where $\varrho_{\alpha \bar{\alpha}}:=H\left(s_{\alpha}, s_{\alpha}\right)$. Formula $(2.5)$ is equivalent to

$$
f=i \varphi\left(\log \varrho_{\bar{\alpha} \alpha}\right)+\varphi_{\alpha}, \quad \varphi_{\alpha} \in \mathcal{O}\left(\Omega_{\alpha}\right) .
$$


Using the transformation rule $\varrho_{\bar{\alpha} \alpha}=g_{\alpha \beta} \bar{g}_{\alpha \beta} \varrho_{\bar{\beta} \beta}$ and (2.6) we find

$$
\varphi\left(\log g_{\alpha \beta}\right)=-i \varphi_{\beta}+i \varphi_{\alpha} \quad \text { on } \quad \Omega_{\alpha} \cap \Omega_{\beta} .
$$

This and $\gamma(f)=\varphi+\bar{\varphi}$ give $\gamma\left(C_{F F}\right) \subset H_{F F}$.

In order to prove the inverse inclusion, let us take $X=\varphi+\bar{\varphi} \in H_{F F}$. By definition, $\varphi$ satisfies (2.7). Thus, there is a function $f \in C^{\infty}(M, \mathbb{C})$ such that (2.6) is satisfied. From

$$
\omega\lrcorner X=-\overline{\partial f}-\partial \bar{f}=-d(\operatorname{Re} f)+i(\partial-\bar{\partial}) \operatorname{Im} f
$$

and from $\mathscr{L}_{X} \omega=0$ we get $\bar{\partial} \partial \operatorname{Im} f=\bar{\partial} \partial\left[\frac{1}{2}(\varphi+\bar{\varphi}) \log \varrho_{\bar{\alpha} \alpha}\right]=0$. The last formula shows that $\frac{1}{2}(\varphi+\bar{\varphi}) \log \varrho_{\bar{\alpha} \alpha}$ is a pluriharmonic function. Thus, (see [19]), there is a $\lambda_{\alpha} \in \mathcal{O}\left(\Omega_{\alpha}\right)$ such that $\frac{1}{2}(\varphi+\bar{\varphi}) \log \varrho_{\bar{\alpha} \alpha}=i\left(\lambda_{\alpha}-\bar{\lambda}_{\alpha}\right)$. From this and from (2.6) we obtain

$$
\operatorname{Im} f=\frac{1}{2 i}\left(2 \lambda_{\alpha}+\varphi_{\alpha}-2 \bar{\lambda}_{\alpha}-\bar{\varphi}_{\alpha}\right) \text { on } \Omega_{\alpha} .
$$

Since $\operatorname{Im} f$ is defined globally, one has $2 \lambda_{\alpha}+\varphi_{\alpha}=2 \lambda_{\beta}+\varphi_{\beta}$ on $\Omega_{\alpha} \cap \Omega_{\beta}$ which in turn defines a holomorphic function $\zeta \in \mathcal{O}(M)$. The cocycle $\left\{\varphi_{\alpha}\right\}$ is given up to a holomorphic function $\xi \in \mathcal{O}(M)$. Therefore, we can choose $\xi$ in such a way that $\zeta=0$, which gives $\operatorname{Im} f=0$. From this and from (2.8) we see that $H_{F F} \subset \gamma\left(C_{F F}\right)$.

Because of Proposition 1 the Kostant-Souriau quantization prescription (2.3) takes in a holomorphic frame the following form:

$$
\hat{f} S=\left[\left(-i \varphi_{\alpha}^{k} \frac{\partial}{\partial z_{\alpha}^{k}}-i \frac{\partial \varphi^{k}}{\partial z_{\alpha}^{k}}+\varphi_{\alpha}\right) \psi_{\alpha}\right] S_{\alpha} \otimes d z_{\alpha}^{1} \wedge \ldots \wedge d z_{\alpha}^{n},
$$

where $s=\psi_{\alpha} s_{\alpha} \otimes d z_{\alpha}^{1} \wedge \ldots \wedge d z_{\alpha}^{n}, \varphi=\varphi_{\alpha}^{k} \frac{\partial}{\partial z_{\alpha}^{k}}$, and $\varphi_{\alpha}, \varphi$ are given by (2.6). Using (2.9) we obtain

$$
d[i H(s, t)-\gamma(f)]=H\left(\hat{f}_{s}, t\right)-H(s, \hat{f} t)
$$

for $f \in C_{F F}$. After integrating both sides of (2.10) and applying Stokes theorem we see that $\hat{f}$ is a symmetric operator on $\mathscr{M}$ if $\partial M=\emptyset$ or if the Hermitian metric $H$ vanishes on $\partial M$.

Fixing of $z \in \Omega_{\alpha}$ and of a holomorphic frame $s_{\alpha} \otimes d z_{\alpha}^{1} \wedge \ldots \wedge d z_{\alpha}^{n}$ enables one to define the evaluation functional $\mathscr{M} \ni s \rightarrow \psi_{\alpha}(z)$, where $\psi_{\alpha}$ are the components of $s$ in the fixed frame. Because of the inequality $\left|\psi_{\alpha}(z)\right| \leqq c_{\alpha}\|S\|$ (see e.g. [3]), where $c_{\alpha}$ is some positive constant depending only on $s_{\alpha} \otimes d z_{\alpha}^{1} \wedge \ldots \wedge d z_{\alpha}^{n}$, this is a continuous linear functional. Thus, by Riesz's theorem, there exists $K_{\bar{\alpha}}(\bar{z}, \cdot)$ such that

$$
\psi_{\alpha}(z)=\left\langle K_{\bar{\alpha}}(\bar{z}, \cdot), s(\cdot)\right\rangle .
$$

From condition (2.2) we have $K_{\bar{\alpha}}(\bar{z}, \cdot) \neq 0$. In the opposite case one has for each $s \in \mathscr{M}$ that $s(z)=0$, which is in contradiction with (2.2). Therefore, taking into account the transformation rule

$$
K_{\bar{\beta}}(\bar{z}, \cdot)=\overline{g_{\alpha \beta}(z)} \overline{\frac{\partial z_{\alpha}}{\partial z_{\beta}}(z)} K_{\bar{\alpha}}(\bar{z}, \cdot),
$$


we obtain, that the following

$$
M \supset \Omega_{\alpha} \ni z \rightarrow\left[K_{\bar{\alpha}}(\bar{z}, \cdot)\right] \in \mathbb{C P}(\mathscr{M})
$$

is a well defined map from the classical phase space $M$ into the quantum phase space $\mathbb{C} \mathbb{P}(\mathscr{M})$.

Writing down $K_{\bar{\alpha}}(\bar{z}, v)$ in a chosen frame,

$$
K_{\bar{\alpha}}(\bar{z}, v)=K_{\bar{\alpha} \beta}(\bar{z}, v) s_{\beta} \otimes d v_{\beta}^{1} \wedge \ldots \wedge d v_{\beta}^{n},
$$

we obtain the reproducing kernel function $K_{\bar{\alpha} \beta}(\bar{z}, v)$ which has the following properties:

a) positivity

$$
K_{\bar{\alpha} \alpha}(\bar{z}, z)>0,
$$

b) reproducing property

$$
K_{\bar{\alpha} \beta}(\bar{z}, v)=\left\langle K_{\bar{\beta}}(\bar{v}, \cdot), K_{\bar{\alpha}}(\bar{z}, \cdot)\right\rangle,
$$

c) transformation rule

$$
K_{\bar{\beta} \gamma}(\bar{z}, v)=\overline{g_{\alpha \beta}(z)} g_{\delta \gamma}(v) \frac{\overline{\partial z_{\alpha}}}{\partial z_{\beta}}(z) \frac{\partial v_{\delta}}{\partial v_{\gamma}}(v) K_{\bar{\alpha} \delta}(\bar{z}, v),
$$

d) the quadratic form

$$
d s^{2}=\sum_{j, k=1}^{n} \frac{\partial^{2} \log K_{\bar{\alpha} \alpha}(\bar{z}, z)}{\partial z^{j} \partial \bar{z}^{k}} d z_{\alpha}^{j} d \bar{z}_{\alpha}^{k}
$$

is invariant under changes of holomorphic frames $s_{\alpha}$ and coordinates $\left(z_{\alpha}^{1}, \ldots, z_{\alpha}^{n}\right)$. These properties will be crucial for the following considerations.

Proposition 2. The following statements are equivalent:

a) $\mathscr{K}: M \rightarrow \mathbb{C P}(\mathscr{M})$ is an injection,

b) for each $z_{1}, z_{2} \in M$ there exist $v_{1}, v_{2} \in M$ such that

$$
\operatorname{det}\left[\begin{array}{ll}
K_{\bar{\gamma} \alpha}\left(\bar{v}_{1}, z_{1}\right), & K_{\bar{\gamma} \beta}\left(\bar{v}_{1}, z_{2}\right) \\
K_{\bar{\delta} \alpha}\left(\bar{v}_{2}, z_{1}\right), & K_{\bar{\delta} \beta}\left(\bar{v}_{2}, z_{2}\right)
\end{array}\right] \neq 0,
$$

where $v_{1} \in \Omega_{\gamma}, v_{2} \in \Omega_{\delta}, z_{1} \in \Omega_{\alpha}$, and $z_{2} \in \Omega_{\beta}$. (The condition (2.17) does not depend on the trivialization of $\mathbb{E} \otimes T^{*(n, 0)} M$.)

c) Condition (2.2) is satisfied.

Proof. If $\mathscr{K}$ is an injection, then for each $z_{1}, z_{2} \in M$ and for $c \in \mathbb{C}^{*}$, we have $K_{\bar{\alpha}}\left(\bar{z}_{1}, \cdot\right)$ $\neq c K_{\bar{\beta}}\left(\bar{z}_{2}, \cdot\right)$. Thus, for each $z_{1}, z_{2} \in M$ and for $c \in \mathbb{C}^{*}$ there exist $v_{1}, v_{2} \in M$ such that

$$
\left[\begin{array}{l}
K_{\bar{\alpha} \gamma}\left(\bar{z}_{1}, v_{1}\right) \\
K_{\bar{\alpha} \delta}\left(\bar{z}_{1}, v_{2}\right)
\end{array}\right] \neq c\left[\begin{array}{l}
K_{\bar{\beta} \gamma}\left(\bar{z}_{2}, v_{1}\right) \\
K_{\bar{\beta} \delta}\left(\bar{z}_{2}, v_{2}\right)
\end{array}\right] .
$$

Because of $\overline{K_{\bar{\alpha} \gamma}(\bar{z}, v)}=K_{\bar{\gamma} \alpha}(\bar{v}, z)$ the last condition is equivalent to (2.17), which gives b). Using (2.15) we find that (2.17) does not depend on the trivialization of $\mathbb{F} \otimes T^{*(n, 0)} M$. Putting $s_{1}=K_{\bar{\gamma}}\left(\bar{v}_{1}, \cdot\right)$ and $s_{2}=K_{\bar{\delta}}\left(\bar{v}_{2}, \cdot\right)$, we obtain c) from the statement b). 
In order to show that c) implies a) let us assume that $\mathscr{K}$ is not an injection. Thus, there exist $z_{1}, z_{2} \in M$ and $c \in \mathbb{C}^{*}$ such that $K_{\bar{\alpha}}\left(\bar{z}_{1}, \cdot\right)=c K_{\bar{\beta}}\left(\bar{z}_{2}, \cdot\right)$. Multiplying the last equality by $s \in \mathscr{M}$ we find $\psi_{\alpha}\left(z_{1}\right)=c \psi_{\beta}\left(z_{2}\right)$. This means that there are $z_{1}, z_{2} \in M$ such that for each $s_{1}, s_{2} \in \mathscr{M}$ we have

$$
\operatorname{det}\left[\begin{array}{ll}
\psi_{1 \alpha}\left(z_{1}\right), & \psi_{1 \beta}\left(z_{2}\right) \\
\psi_{2 \alpha}\left(z_{1}\right), & \psi_{2 \beta}\left(z_{2}\right)
\end{array}\right]=0
$$

which is in contradiction with c).

Proposition 3. The map $\mathscr{K}: M \rightarrow \mathbb{P C}(\mathscr{M})$ is an anti-holomorphic embedding if and only if the condition (2.17) is satisfied and $d s^{2}$ is positive definite (in this case it is called the Bergman metric).

Proof. The proof stated here is a simple extension of the proof contained in [8] to the case of general line bundle. Let $\mathscr{M}_{z}=\{\psi \in \mathscr{M}: \psi(z)=0\}$ and let $\mathscr{M}_{z}^{\perp}$ be the orthogonal complement of $\mathscr{M}_{z}$ in $\mathscr{M}$. From the condition (2.2) one has: $\operatorname{dim}_{\mathbb{C}} \mathscr{M}_{z}^{\perp}=1$. Let us choose an orthonormal base $\left\{s_{0}, s_{1}, s_{2}, \ldots\right\}$ in $\mathscr{M}$ consistent with the decomposition $\mathscr{M}=\mathscr{M}_{z} \oplus \mathscr{M}_{z}^{\perp}$, i.e. $\left\langle s_{1}, s_{2}, \ldots\right\rangle=\mathscr{M}_{z}$ and $\left\langle s_{0}\right\rangle=\mathscr{M}_{z}^{\perp}$. Taking $K_{\bar{\alpha}}(\bar{z}, \cdot)=\sum_{n=0}^{\infty} \kappa_{\bar{\alpha} n}(\bar{z}) s_{n}$ and $s_{n}=\Psi_{n \alpha} S_{\alpha} \otimes d z_{\alpha}^{1} \wedge \ldots \wedge d z_{\alpha}^{2}$, one concludes from the reproducing property that $\kappa_{\bar{\alpha} n}(\bar{z})=\overline{\psi_{n \alpha}(z)}$, which gives

$$
d s_{z}^{2}=\sum_{n=1}^{\infty} \frac{d \kappa_{\bar{\alpha} n}(\bar{z}) \overline{d \kappa_{\bar{\alpha} n}(\bar{z})}}{\left|\kappa_{\bar{\alpha}_{0}}(\bar{z})\right|^{2}} .
$$

From (2.18) one sees that $d s^{2}$ is positive definite at $z \in M$ if and only if for every $\varphi_{z} \in T_{z}^{(1,0)} M$ there exists $s \in \mathscr{M}_{z}$ such that $\varphi_{z}\left(\psi_{\alpha}\right) \neq 0$, where $s=\psi_{\alpha} s_{\alpha} \otimes d z_{\alpha}^{1} \wedge \ldots \wedge d z_{\alpha}^{n}$. But the last condition strictly means that the differential $d \mathscr{K}$ of $\mathscr{K}: M \rightarrow \mathbb{P C}(\mathscr{M})$ is nonsingular. Due to this and from Proposition 2 we have that $\mathscr{K}$ is an embedding. If one assumes that $\mathscr{K}$ is an embedding then according to Proposition 2 the condition (2.17) is satisfied. For $d s^{2}$ one has $d s^{2}=\overline{\mathscr{K}}^{*} d s_{F S}^{2}$, where $d s_{F S}^{2}$ is the FubiniStudy metric on $\mathbb{P C}(\mathscr{M})$. Thus $d s^{2}$ is positive definite.

The above propositions show that the positive definiteness of $d s^{2}$ and (2.17) ensure that the classical phase space is embedded into the quantum phase space. In this manner, we can quantize not only observables $f \rightarrow \hat{f}$ but also the states $\mathscr{K}: z \rightarrow\left[K_{\bar{\alpha}}(\bar{z}, \cdot)\right]$. In the case of the Bargmann-Fock representation $\left[K_{\bar{\alpha}}(\bar{z}, \cdot)\right]$ is a so-called coherent state; hence we extend this terminology to the general case. The reproducing property guarantees that coherent states form a linearly dense subset in $\mathscr{M}$.

Also some other properties of the reproducing kernel $\left\{K_{\bar{\alpha} \beta}(\bar{z}, v)\right\}$ are to be mentioned here. From (2.15) we see that it is a section of the line bundle $\operatorname{pr}_{1}^{*}\left(\overline{\mathbb{E}} \otimes T^{*(0, n)} M\right) \otimes \operatorname{pr}_{2}^{*}\left(\mathbb{E} \otimes T^{*(n, 0)} M\right)$, where $\operatorname{pr}_{i}$ is the projection of $M \times M$ on the $i^{\text {th }}$ component. Thus, the diagonals $\left\{K_{\bar{\alpha} \alpha}(\bar{z}, z)\right\}$ define a section of $\overline{\mathbb{E}} \otimes \mathbb{E} \otimes T^{*(n, n)} M . \quad$ The transformation rule $\omega_{\bar{\beta} \beta}=\omega_{\bar{\alpha} \alpha} \frac{\overline{\partial z_{\alpha}}}{\partial z_{\beta}} \frac{\partial z_{\alpha}}{\partial z_{\beta}}$, where $\omega_{\bar{\alpha} \alpha}=\operatorname{det}\left[\omega_{j \bar{k}}\right] \neq 0$ is a component of the Liouville $2 n$-form $\bigwedge_{\wedge}^{n} \omega$ 
$=-n ! \omega_{\bar{\alpha} \alpha} d \bar{z}_{\alpha}^{1} \wedge \ldots \wedge d \bar{z}_{\alpha}^{n} \wedge d z_{\alpha}^{1} \wedge \ldots \wedge d z_{\alpha}^{n}$, gives

$$
\frac{K_{\bar{\beta} \beta}(\bar{z}, z)}{(-i)^{n} \omega_{\bar{\beta} \beta}(z)}=\left|g_{\alpha \beta}(z)\right|^{2} \frac{K_{\bar{\alpha} \alpha}(\bar{z}, z)}{(-i)^{n} \omega_{\bar{\alpha} \alpha}(z)} \quad \text { for } \quad z \in \Omega_{\alpha} \cap \Omega_{\beta} .
$$

This means that $\frac{(-i)^{n} \omega_{\bar{\alpha} \alpha}}{K_{\bar{\alpha} \alpha}}$ are components of some section $\tilde{H}$ of the bundle $\overline{\mathbb{E}}^{*} \otimes \mathbb{E}^{*}$ written in the frame $\bar{s}_{\alpha} \otimes s_{\alpha}$. Because $\omega$ is nonsingular we have $\frac{(-i)^{n} \omega_{\bar{\alpha} \alpha}}{K_{\bar{\alpha} \alpha}} \neq 0$. Therefore, $\tilde{H}$ or $-\tilde{H}\left(\right.$ when $\frac{(-i)^{n} \omega_{\bar{\alpha} \alpha}}{K_{\bar{\alpha} \alpha}}$ is negative $)$ defines a new metric structure on $\mathbb{E}$. Everywhere below we shall assume the positivity of $\frac{(-i)^{n} \omega_{\bar{\alpha} \alpha}}{K_{\bar{\alpha} \alpha}}$.

The physical meaning of $K_{\bar{\alpha} \beta}(\bar{z}, v)$ is determined by the following. Identifying the classical states $z \in \Omega_{\alpha}$ and $v \in \Omega_{\beta}$ with the coherent quantum states $\left[K_{\bar{\alpha}}(\bar{z}, \cdot)\right]$ and $\left[K_{\bar{\beta}}(\bar{v}, \cdot)\right]$ respectively, we can calculate the transition probability amplitude

$$
a_{\bar{\beta} \alpha}(\bar{v}, z):=\left\langle\frac{K_{\bar{\alpha}}(\bar{z}, \cdot)}{\left\|K_{\bar{\alpha}}(\bar{z}, \cdot)\right\|}, \frac{K_{\bar{\beta}}(\bar{v}, \cdot)}{\left\|K_{\bar{\beta}}(\bar{v}, \cdot)\right\|}\right\rangle=\frac{K_{\bar{\beta} \alpha}(\bar{v}, z)}{K_{\bar{\beta} \beta}(\bar{v}, v)^{1 / 2} K_{\bar{\alpha} \alpha}(\bar{z}, z)^{1 / 2}} .
$$

After passing to a new frame [see transformation rule (2.15)] $a_{\bar{\beta} \alpha}(\bar{v}, z)$ changes only by a phase factor. Hence, the transition probability density $\left|a_{\bar{\beta} \alpha}(\bar{v}, z)\right|^{2}$ does not depend on a choice of the bundle trivialization, but it naturally depends on points $z \in \Omega_{\alpha}$ and $v \in \Omega_{\beta}$. The transition probability amplitude from $z$ to $v$ with the simultaneous transition through the point $w \in \Omega_{\gamma}$ is $a_{\bar{\gamma} \alpha}(\bar{w}, z) a_{\bar{\beta} \gamma}(\bar{v}, w)$, and it also does not depend on the choice of frame $s_{\gamma}: \Omega_{\gamma} \rightarrow \mathbb{E}$. Rephrasing the reproducing property (2.14) in terms of the transition probability amplitude we obtain

$$
a_{\bar{\beta} \alpha}(\bar{v}, z)=\sum_{\gamma} \int_{M} a_{\bar{\gamma} \alpha}(\bar{w}, z) a_{\bar{\beta} \gamma}(\bar{v}, w) K_{\bar{\gamma} \gamma}(\bar{w}, w) \varrho_{\bar{\gamma} \gamma}(w) h_{\gamma}(w) d w_{\gamma}^{1} \wedge \ldots \wedge d \bar{w}_{\gamma}^{n}
$$

where supp $h_{\gamma} \subset \Omega_{\gamma}, \sum_{\gamma} h_{\gamma}=1$, and $h_{\gamma} \in C^{\infty}(M, \mathbb{R})$, i.e. $\left\{h_{\gamma}\right\}$ is a partition of unity. The natural measure on the phase space is the Liouville measure

$$
d \mu_{L}:=(-i)^{n} \omega_{\bar{\gamma} \gamma} d w_{\gamma}^{1} \wedge \ldots \wedge d w_{\gamma}^{n} \wedge d \bar{w}_{\gamma}^{1} \wedge \ldots \wedge d \bar{w}_{\gamma}^{n} .
$$

It is then physically reasonable to perform an integration in (2.21) with respect to $d \mu_{L}$ instead of $\varrho_{\bar{\gamma} \gamma}(w) K_{\bar{\gamma} \gamma}(\bar{w}, w) d w_{\gamma}^{1} \wedge \ldots \wedge d \bar{w}_{\gamma}^{n}$. Therefore, let us assume

$$
d \mu_{L}(w)=C \varrho_{\bar{\gamma} \gamma}(w) K_{\bar{\gamma} \gamma}(\bar{w}, w) d w_{\gamma}^{1} \wedge \ldots \wedge d w_{\gamma}^{n} \wedge d \bar{w}_{\gamma}^{1} \wedge \ldots \wedge d \bar{w}_{\gamma}^{n},
$$

where $0<C$, which means that the metric $\tilde{H}$ coincides, up to a real positive constant, with the metric $H, \widetilde{H}=C H$. Locally the above condition takes the form of the complex Monge-Ampère equation

$$
\operatorname{det}\left[\frac{\partial^{2} \log \varrho_{\bar{\gamma} \gamma}(w)}{\partial w_{j} \partial \bar{w}_{k}}\right]=C(-1)^{\frac{n(n+1)}{2}} \frac{1}{n !} \varrho_{\bar{\gamma} \gamma}(w) K_{\bar{\gamma} \gamma}(\bar{w}, w) \text { on } \Omega_{\gamma} .
$$

In this way we obtain a differential equation for $\varrho_{\bar{\gamma} \gamma}=H\left(s_{\gamma}, s_{\gamma}\right)$, because $\left\{K_{\bar{\gamma} \gamma}\right\}$ depends on the holomorphic and metric structure of $\mathbb{E}$. In [12] one shows that in 
the case of $M$ being biholomorphic with the bounded domain in $\mathbb{C}^{n},\left\{K_{\bar{\alpha} \beta}\right\}$ depends on $\left\{\varrho_{\bar{\gamma} \gamma}\right\}$ analytically.

Integration of the complex Monge-Ampère equation according to its nonlinear character is a difficult problem, and the discussion of it goes beyond this paper. Significant progress in this direction has been made by Yau (see [20]). In the following sections we consider some cases for which one can find solutions of (2.22).

Now, in order to describe our model in the language of path integrals we shall calculate the transition probability amplitude along the path $\gamma$ for the physical system defined by the phase space $M$. We shall treat this path as a piecewise smooth curve in $M$, beginning at $z$ and ending at $v$. Let $z_{i}=\gamma\left(\tau_{i}\right), i=1, \ldots, N$, be a sequence of points on $\gamma$ such that $z_{1}=z$ and $z_{N}=v$. The transition probability amplitude from the state $z$ to the state $v$ with its simultaneous transition through the states $z_{2}, \ldots, z_{N-1}$, according to the amplitude multiplication rule, will be equal to $\prod_{i=1}^{N-1} a_{\bar{\alpha}_{i+1} \alpha_{i}}\left(\bar{z}_{i+1}, z_{i}\right)$. With the division $\left\{z_{i}\right\}$ of $\gamma$ getting denser and denser we shall find the formula for the transition probability amplitude along $\gamma$,

$$
a_{\bar{\beta} \alpha}(\gamma ; \bar{v}, z)=\lim _{N \rightarrow \infty} \exp \sum_{i=1}^{N-1} \log a_{\bar{\alpha}_{i+1} \alpha_{\imath}}\left(\bar{z}_{i+1}, z_{i}\right),
$$

where $\alpha=\alpha_{1}, \beta=\alpha_{N}$, and $z_{i} \in \Omega_{\alpha_{i}}$. Hence, because of $K_{\bar{\alpha}_{i+1} \alpha_{l}}\left(\bar{z}_{i+1}, z_{i}\right)$ are differentiable functions of their arguments, we obtain

$$
a_{\bar{\beta} \alpha}(\gamma ; \bar{v}, z)=\exp \left[i \int_{\gamma} \operatorname{Im}(\bar{\partial} \log K)\right],
$$

where the 1 -form $\operatorname{Im}(\bar{\partial} \log K)$ is given locally by $\operatorname{Im}\left(\bar{\partial} \log K_{\bar{\gamma} \gamma}\right)$. The formula (2.24) has a geometrical interpretation. Namely, one can see from $(2.15)$ that $K_{\bar{\gamma} \gamma}(\bar{z}, z)^{-1}$ can be considered as the component of some Hermitian metric $H^{K}$ on the bundle $\mathbb{E} \otimes T^{*(n, 0)} M$ taken in the holomorphic frame $s_{\gamma} \otimes d z_{\gamma}^{1} \wedge \ldots \wedge d z_{\gamma}^{n}$,

$$
K_{\bar{\gamma} \gamma}(\bar{z}, z)^{-1}=: H^{K}\left(s_{\gamma} \otimes d z_{\gamma}^{1} \wedge \ldots \wedge d z_{\gamma}^{n}, s_{\gamma} \otimes d z_{\gamma}^{1} \wedge \ldots \wedge d z_{\gamma}^{n}\right) .
$$

Let us take the unitary frame

$$
\sigma_{\gamma}=K_{\bar{\gamma} \gamma}(\bar{z}, z)^{1 / 2} s_{\gamma} \otimes d z_{\gamma}^{1} \wedge \ldots \wedge d z_{\gamma}^{n}, \quad H^{K}\left(\sigma_{\gamma}, \sigma_{\gamma}\right)=1 .
$$

We have

$$
\nabla^{K} \sigma_{\gamma}=i \operatorname{Im}\left(\bar{\partial} \log K_{\bar{\gamma} \gamma}^{-1}\right) \otimes \sigma_{\gamma},
$$

where $\nabla^{K}$ is the metric connection on $\mathbb{E} \otimes T^{*(n, 0)} M$ defined by $H^{K}$. From this we conclude that $a_{\bar{\beta} \alpha}(\gamma ; \bar{v}, z)$ is the parallel transport with respect to the connection $\nabla^{K}$ from point $z$ to point $v$ along the curve $\gamma$. As a consequence of this and (2.15) we find that after passing to a new frame, $a_{\bar{\beta} \alpha}(\gamma ; \bar{v}, z)$ changes by the phase factor which depends on $z$ and $v$ only.

Let $K_{2}, \ldots, K_{N-1}$ be a sequence of regions in $M$ such that $\gamma\left(\tau_{i}\right)=z_{i} \in K_{i}$. Then, according to the rules of superposition and multiplication of the transition probability amplitudes the formula

$$
\begin{aligned}
a_{\bar{\beta} \alpha}\left(\bar{v}, z ; K_{2}, \ldots, K_{N-1}\right):= & \int_{K_{2}} d \mu_{L}\left(z_{2}\right) \ldots \int_{K_{N-1}} d \mu_{L}\left(z_{N-1}\right) \\
& \times a_{\bar{\alpha} \alpha}\left(\bar{z}_{2}, z\right) \ldots a_{\bar{\beta} \alpha_{N-1}}\left(\bar{v}, z_{N-1}\right),
\end{aligned}
$$


where for simplicity we assume $K_{i} \subset \Omega_{\alpha_{i}}$, describes the transition probability amplitude from state $z$ to state $v$ with a simultaneous transition through "the gates" $K_{2}, \ldots, K_{N-1}$. While using the path integral formulation, we assume

$$
a_{\bar{\beta} \alpha}\left(\bar{v}, z ; K_{2}, \ldots, K_{N-1}\right)=: \int_{\mathscr{K}_{N}} \mathscr{D}[\gamma] \exp \left[i \int_{\gamma} \operatorname{Im}(\bar{\partial} \log K)\right]
$$

to be the definition of the path integral of the functional exp $\left[i \int_{\gamma} \operatorname{Im}(\bar{\partial} \log K)\right]$ over the set $\mathscr{K}_{N}$, where $\mathscr{K}_{N}$ is the set of paths which satisfy: $\gamma\left(\tau_{1}\right)=z, \gamma\left(\tau_{i}\right) \in K_{i}$, and $\gamma\left(\tau_{N}\right)=v$. In the special case we will have

$$
a_{\bar{\beta} \alpha}(\bar{v}, z)=: \int \mathscr{D}[\gamma] \exp \left[i \int_{\gamma} \operatorname{Im}(\bar{\partial} \log K)\right] .
$$

Here we integrate over all paths which connect points $z$ and $v$.

The standard metric on projective Hilbert space $\mathbb{C P}(\mathscr{M})$,

$$
d\left(\left[s_{1}\right],\left[s_{2}\right]\right):=\inf _{t_{1}, t_{2} \in \mathbb{R}}\left\|\frac{e^{i t_{1}} s_{1}}{\left\|s_{1}\right\|}-\frac{e^{i t_{2}} s_{2}}{\left\|s_{2}\right\|}\right\|,
$$

can be transported onto the classical phase space $M$ by the imbedding $\mathscr{K}: M \rightarrow \mathbb{C P}(\mathscr{M})$. Putting $s_{1}=K_{\bar{\alpha}}\left(\bar{z}_{1}, \cdot\right)$ and $s_{2}=K_{\bar{\beta}}\left(\bar{z}_{2}, \cdot\right), z_{1} \in \Omega_{\alpha}$, and $z_{2} \in \Omega_{\beta}$, after simple calculation, we obtain

$$
d_{M}\left(z_{1}, z_{2}\right)=\sqrt{2}\left(1-\left|a_{\bar{\alpha} \beta}\left(\bar{z}_{1}, z_{2}\right)\right|\right)^{1 / 2} .
$$

The properties of $d_{M}$ in the case when $M$ is a bounded domain in $\mathbb{C}^{n}$ are studied, e.g. in [17]. It is shown there that the topologies induced by $d_{M}$ and by the Euclidean metric are the same, and $M$ is complete with respect to $d_{M}$. From (2.30) we can see for example that the probability of transition from $z_{1}$ to $z_{2}$ is nearly 1 if $z_{1}$ is close to $z_{2}$ in the sense of the metric $d_{M}$. The sequence of states $\left\{z_{N}\right\}$ of the physical system is a Cauchy sequence if starting from a certain natural number $\mathscr{N}$ the probability of the successive transitions $z_{N} \rightarrow z_{N+1}$ is arbitrarily close to 1 . In other words the most probable physical processes are those which are described by the Cauchy sequences.

\section{Deformation of the Holomorphic and Metric Structure of the Quantum Bundle and Interaction}

One of the ideas of Penrose's twistor theory is the description of electromagnetic and gravitational interactions by the deformation of the holomorphic structure of some complex bundles over twistor flag spaces (see [13,14]). The fruitfulness of such an approach for classical field theory was manifested in the problem of classification of the instanton solutions of Yang-Mills equations (see [1]). Making use of this idea, we fix the differential structure of the quantum bundle $\mathbb{E} \rightarrow M$ and vary its holomorphic and metric structures, interpreting them as external fields interacting with the physical system described by $M$. The purpose of this section is to show how the action functional of the system depends on the above mentioned structures. In order to do this we will compare (2.28) with the Feynman definition of the transition probability amplitude along the path. 
We shall start with the presentation of some necessary facts concerning the spaces of all possible holomorphic and metric structures of the complex differential bundle $\mathbb{E}$. First of all, let us recall that holomorphic line bundles on the complex differential manifold $M$ are, up to isomorphism, described by elements of $H^{1}\left(M, \mathcal{O}^{*}\right)$ (see e.g. [4]). The exact sequence of sheaves

$$
0 \rightarrow \mathbb{Z} \stackrel{\imath}{\longrightarrow} \mathcal{O} \stackrel{\exp }{\longrightarrow} \mathcal{O}^{*} \rightarrow 0
$$

defines the boundary homomorphism $\delta: H^{1}\left(M, \mathcal{O}^{*}\right) \rightarrow H^{2}(M, \mathbb{Z})$ (see e.g. [4]). Thus, because the differential structure of $\mathbb{E}$ has been fixed, the classes of isomorphic holomorphic structures on $\mathbb{E}$ are parametrized by $\delta^{-1}\left(c_{1}(\mathbb{E})\right)$, where $c_{1}(\mathbb{E})$ is the Chern class of $\mathbb{E}$. For a more explicit description, let us now fix a holomorphic structure on $\mathbb{E}$ and let $g_{\alpha \beta}^{0} \in \mathcal{O}^{*}\left(\Omega_{\alpha} \cap \Omega_{\beta}\right)$ and $s_{\alpha}^{0}: \Omega_{\alpha} \rightarrow \mathbb{E}$ be the transition functions and holomorphic frames of it respectively (we will assume that $\left\{\Omega_{\alpha}\right\}$ is an acyclic cover of $M$ ). Let us also fix a Hermitian metric $H^{0}$ on $\mathbb{E}$. Now, for any holomorphic structure of $\mathbb{E}$, which is trivialized by the system of frames $s_{\alpha}: \Omega_{\alpha} \rightarrow \mathbb{E}, s_{\alpha}=g_{\alpha \beta} s_{\beta}$, one has $s_{\alpha}=f_{\alpha} s_{\alpha}^{0}$, where $f_{\alpha} \in C^{\infty}\left(\Omega_{\alpha}\right)$. Any metric structure $H$ on $\mathbb{E}$ is given by $H=\varrho H^{0}$, where $\varrho \in C^{\infty}\left(M, \mathbb{R}^{+}\right)$. Therefore, the system of the smooth functions $\left\{f_{\alpha}\right\}$ taken up to the holomorphic factors $g_{\alpha} \in \mathcal{O}^{*}\left(\Omega_{\alpha}\right)$ and satisfying the transformation rules $f_{\beta}=\frac{g_{\alpha \beta}}{g_{\alpha \beta}^{0}} f_{\alpha}$ on $\Omega_{\alpha} \cap \Omega_{\beta}$ describes the possible holomorphic structures of $\mathbb{E}$. On the other hand the possible metric structures are determined by positive valued smooth functions on $M$. One has

$$
\varrho_{\bar{\alpha} \alpha}=\varrho\left|f_{\alpha}\right|^{2} \varrho_{\bar{\alpha} \alpha}^{0},
$$

where $\varrho_{\bar{\alpha} \alpha}=H\left(s_{\alpha}, s_{\alpha}\right)$ and $\varrho_{\bar{\alpha} \alpha}^{0}=H^{0}\left(s_{\alpha}^{0}, s_{\alpha}^{0}\right)$. For the later application, it is reasonable to introduce the following notation

$$
e^{B_{\alpha}}:=\varrho\left|f_{\alpha}\right|^{2} \quad \text { and } \quad e^{A_{\alpha}}:=\frac{K_{\bar{\alpha} \alpha}}{K_{\bar{\alpha} \alpha}^{0}} .
$$

The functions $A_{\alpha}: \Omega_{\alpha} \rightarrow \mathbb{R}$ describe the reproducing kernel deformation resulting from the deformation $B_{\alpha}: \Omega_{\alpha} \rightarrow \mathbb{R}$ of the holomorphic and metric structures.

The dependence of $\left\{A_{\alpha}\right\}$ on $\left\{B_{\alpha}\right\}$ plays the fundamental role in our theory. The effective use of it demands the formulas that would explicitly express the potentials $A_{\alpha}=A_{\alpha}(\bar{z}, z)$ in terms of $B_{\alpha}=B_{\alpha}(\bar{z}, z)$. The search for such formulas is equivalent to the calculation of the reproducing kernel functions $K_{\bar{\alpha} \alpha}(\bar{z}, z)$, which is in the general case an unsolved problem. However, the result obtained in [12] suggests that in the case of a small deformation (i.e. when $B_{\alpha}$ is small) the perturbative methods can be used in the analysis of $A_{\alpha}=A_{\alpha}\left(\left\{B_{\beta}\right\}\right)$. In view of the dependence $A_{\alpha}=A_{\alpha}\left(\left\{B_{\beta}\right\}\right)$ the equation

$$
\operatorname{det}\left[\frac{\partial^{2} \log \varrho_{\bar{\alpha} \alpha}^{0}}{\partial z_{\alpha}^{j} \partial \bar{z}_{\alpha}^{k}}+\frac{\partial^{2} B_{\alpha}}{\partial z_{\alpha}^{j} \partial \bar{z}_{\alpha}^{k}}\right]=\operatorname{det}\left[\frac{\partial^{2} \log \varrho_{\alpha \alpha}^{0}}{\partial z_{\alpha}^{j} \partial \bar{z}_{\alpha}^{k}}\right] e^{B_{\alpha}+A_{\alpha}\left(\left\{B_{\beta}\right\}\right)}
$$

which is the consequence of imposing condition $(2.22)$ on $\left(s_{\alpha}^{0}, \varrho_{\bar{\alpha} \alpha}^{0}\right)$ and $\left(s_{\alpha}, \varrho_{\bar{\alpha} \alpha}\right)$, can be treated as a field equation for the field $\left\{B_{\beta}\right\}$. If $\left\{B_{\beta}\right\}$ is a solution then $\left\{A_{\alpha}\right\}$ can be obtained from (3.3). 
In order to simplify further considerations we shall study the case with the existence of coordinates $\left(z^{1}, \ldots, z^{n}\right)$ on the domain $\Omega \subset M$ which covers $M$ up to the zero measure set. We will also assume that there exist holomorphic frames $s^{0}: \Omega \rightarrow \mathbb{E}$ and $s: \Omega \rightarrow \mathbb{E}$ corresponding to holomorphic structures defined by the 1-cocycles $\left\{g_{\alpha \beta}^{0}\right\}$ and $\left\{g_{\alpha \beta}\right\}$ respectively. From now on all quantities appearing above will be denoted by the same symbols and without the indices enumerating the frames. Let us now take the $\operatorname{map} \Omega \ni z \rightarrow K(\bar{z}, \cdot) \in \mathscr{M}$. This allows us to consider $z$ as an element of a Hilbert space and in such a way to calculate its norm $\|K(\bar{z}, \cdot)\|$ $=K(\bar{z}, z)^{1 / 2}$. Hence, a unitary process $\left[\tau_{i}, \tau_{f}\right] \ni \tau \rightarrow z(\tau)=K(\bar{z}(\tau), \cdot) \in \mathscr{M}$ satisfies the condition $K(\bar{z}(\tau), z(\tau))=k_{0}=$ const. Generally one can take

$$
f\left[\frac{1}{k_{0}} K(\bar{z}(\tau), z(\tau))\right]=f_{0}=\text { const },
$$

where $f$ is a one-to-one real smooth function on $\mathbb{R}^{+}$. The examples presented below show that for many reasons it is reasonable to put $f=\log$.

Let us then calculate the transition probability amplitude $a(\bar{w}, z)$ however, under the condition that the transitions of the system from $z$ to $w$ will be realized by the unitary processes $z(\tau)$, i.e. when the restriction (3.4) is taken into account and $z\left(\tau_{i}\right)=z, z\left(\tau_{f}\right)=w$. Unitarity of the process $\tau \rightarrow K(\bar{z}(\tau), \cdot)$ is a quantum states phase space counterpart of the energy conservation law which is assured in the classical phase space $M$, while unitarity in $\mathscr{M}$ is assumed.

Using the standard arguments and (2.28) we obtain

$$
\begin{aligned}
a\left(\bar{w}, z ; k_{0}\right)= & \int \prod_{\tau} d z(\tau) d \bar{z}(\tau) \delta\left[f\left(\frac{1}{k_{0}} K(\bar{z}(\tau), z(\tau))\right)-f(1)\right] \exp [i \operatorname{Im}(\bar{\partial} \log K)] \\
= & \int \prod_{\tau} d z(\tau) d \bar{z}(\tau) d \lambda(\tau) \exp \frac{i}{h}\left\{\int _ { \tau _ { i } } ^ { \tau _ { f } } h \left[\frac{1}{2 i}\left(\frac{\partial \log K}{\partial \bar{z}^{k}} \frac{d \bar{z}^{k}}{d \tau}-\frac{\partial \log K}{\partial z^{k}} \frac{d z^{k}}{d \tau}\right)\right.\right. \\
& \left.\left.+\lambda(\tau)\left(f\left(\frac{1}{k_{0}} K(\bar{z}(\tau), z(\tau))\right)-f(1)\right)\right] d \tau\right\} .
\end{aligned}
$$

where $\lambda(\tau), \tau \in\left[\tau_{i}, \tau_{f}\right]$ are the Lagrange multipliers resulting from (3.4). Integration over $d \lambda(\tau)$ respects the contribution to functional integral given by all the possible parametrizations of the considered processes. Fixing $\lambda(\tau)$, it is natural to treat $t=\int_{t_{0}}^{t} \lambda(\tau) d \tau$ as the time parameter, measured by some classical measuring instrument - the clock. Therefore, taking into account the Feynman definition of the transition probability amplitude along the path, we will define the action functional for the system as

$$
S[\gamma]=\int_{\gamma} d L:=h \int_{t_{i}}^{t_{f}} \operatorname{Im}\left(\bar{\partial} \log K_{0}\right)+\left[\operatorname{Im} \frac{\bar{\partial} A}{d t}+f\left(\frac{1}{k_{0}} K\right)\right] d t .
$$

While defining $S$ we substitute $K(\bar{z}, z)=K_{0}(\bar{z}, z) e^{A(\bar{z}, z)}$ and neglect the phase factor $\exp \left[i\left(t_{f}-t_{i}\right) f(1)\right]$, which has no meaning in the considerations presented here. The expressions $\operatorname{Im}\left(\bar{\partial} \log K_{0}\right)$ and $\operatorname{Im} \frac{\bar{\partial} A}{d t}+f\left(\frac{1}{k_{0}} K\right)$ will be interpreted as the oneform responsible for the Legendre transformation and the Hamiltonian of the 

considered system. According to the previous interpretation of $A$ the term $\operatorname{Im} \frac{\bar{\partial} A}{d t}$
ensures the interaction of the system with the external field.

As an illustration we will consider the following:

Example. Let us take $M=\mathbb{C}^{n}$ and $\mathbb{E}=\mathbb{C}^{n} \times \mathbb{C}$ as the quantum bundle with the Hermitian metric

$$
\varrho^{0}=H\left(s^{0}, s^{0}\right)=\exp \left[-\frac{1}{2 h}\left(\left|z^{1}\right|^{2}+\ldots+\left|z^{n}\right|^{2}\right)\right],
$$

where the holomorphic frame $s^{0}: \mathbb{C}^{n} \rightarrow \mathbb{E}$ is given by the $s^{0}(z):=z \times\{1\}$ and $h>0$. After a simple calculation one finds the reproducing kernel function

$$
K_{0}(\bar{v}, z)=(2 \pi h)^{-n} \exp \left[\frac{1}{2 h}\left(\bar{v}^{1} z^{1}+\ldots+\bar{v}^{n} z^{n}\right)\right] .
$$

The function $\varrho^{0}$ satisfies the condition $(2.22)$ for $C=(-1)^{\frac{n(n+3)}{2}} n$ !. Taking $z^{k}=x^{k}+i y^{k}, f=\log$ and $A=0$ (i.e. the external field is zero) one obtains from (3.6) the action functional

$$
S[\gamma]=\frac{1}{h} \int_{t_{i}}^{t_{f}} p^{k} d x^{k}-\frac{1}{2}\left(p^{k} p^{k}+x^{k} x^{k}\right) d t-\left.\frac{1}{2 h} p^{k} x^{k}\right|_{t_{i}} ^{t_{f}}-\left(t_{f}-t_{i}\right) \log k_{0}
$$

for the $n$-dimensional isotropic harmonic oscillator. As a consequence (3.5) gives the Feynman propagator for the harmonic oscillator. The quantization procedure, described in Sect. 2, leads to the Bargmann-Fock-Segal quantization for the physical quantities and replaces the classical state $v$ by the corresponding coherent state $(2 \pi h)^{-n} \exp \left[\frac{1}{2 h} \bar{v}^{k} z^{k}\right]$.

The variation principle $\frac{\delta S}{\delta \gamma}=0$ for (3.6) leads to the following Hamiltonian equations:

$$
\left.\omega^{K}\right\lrcorner X=-d f\left(\frac{1}{k_{0}} K\right)
$$

where the $(1,1)$-form $\omega^{K}=i \bar{\partial} \partial \log K$ is the curvature form of metric connection $\nabla^{K}$ and $X=\frac{d z^{k}}{d t} \frac{\partial}{\partial z^{k}}+\frac{d \bar{z}^{k}}{d t} \frac{\partial}{\partial \bar{z}^{k}}$. The choice of the function $f$ influences only the time parameter rescaling.

To end this section let us make a few remarks on the invariance of the presented theory with respect the holomorphic gauge transformation, given by replacement of the holomorphic frame $s: \Omega \rightarrow \mathbb{E}$ by $s^{\prime}=g s$, where $g \in \mathcal{O}^{*}(\Omega)$. The transformation rule (2.15) shows that the transition probabilities $|a(\bar{v}, z)|^{2},\left|a\left(\bar{v}, z ; K_{1}, \ldots, K_{N-1}\right)\right|^{2}$, $|a(\gamma ; \bar{v}, z)|^{2}$, and the metric $d_{M}$ are gauge invariants. Also the Monge-Ampère condition possesses this property. On the other hand, since, one has

$$
K^{\prime}(\bar{z}(\tau), z(\tau))=|g(z(\tau))|^{2} K(\bar{z}(\tau), z(\tau)),
$$


where $K^{\prime}(\bar{z}, z)$ is the reproducing kernel function taken in the new frame $s^{\prime}$, the unitarity property (3.4) is lost after passing to the new gauge. Therefore, the problem of physical meaning of the holomorphic gauge arises. We suppose that its choice should be made in each individual case independently. So, it is reasonable not to discuss this problem generally.

\section{The Application to the Scalar Massive Conformal Particle Case}

In this section we apply the formalism developed in two previous sections to the phase space $\mathbb{M}^{++}$of all possible "creations of classical scalar massive particle." By "creations of classical scalar massive particle" we mean the separate effects of identification of what is called an object, localized in space-time with momentum and with both positive energy and mass. We shall not fix the mass, therefore, $\mathbb{M}^{++}$ is an 8-dimensional manifold parametrized by four spacetime coordinates and four-momenta. We shall assume also that $\mathbf{M}^{++}$is an $S U(2,2)$-homogeneous symplectic manifold. The conformal group $S U(2,2)$ is a natural extension of the Poincaré group by dilatation and four-acceleration transformations. If we want to obtain the phase space for the relativistic scalar massive particle, which could be described as some trajectory in $\mathbf{M}^{++}$localized on a constant mass hypersurface, we should reduce the symplectic structure of $\mathbb{M}^{++}$to the mass-shell. Hence, it is natural to call the "creation of scalar massive particle" the conformal scalar massive particle.

It was shown in [11] that one of the possible realizations of $\mathbb{M}^{++}$is the Grassmannian of two-dimensional positive definite complex subspaces in twistor space $\mathbb{T}$. Let us recall that $\mathbb{T}$ by definition is $\mathbb{C}^{4}$ equipped with a Hermitian form $\eta$ with signature ++-- , and a two-dimensional subspace $z \in G(2, \mathbb{T})=: \mathbb{M}$ is positive definite iff the twistor form $\eta$ restricted to $z$ is positive definite, i.e. sign $\left.\eta\right|_{z}=++$. The conformal compactification of Minkowski space is the Grassmannian $\mathbb{M}^{00}$ of isotropic (with respect to $\eta$ ) two-subspaces in $\mathbb{T}$. $\mathbb{M}^{00}$ and $\mathbb{M}^{++}$ are conformally homogeneous spaces. One can consider $S U(2,2) / \mathbb{Z}_{4}$ as the biholomorphism group for $\mathbb{M}^{++}$. The complexification of $\mathbb{M}^{00}$ gives $\mathbb{M}$ and $\mathbb{M}^{00} \subset \partial \mathbb{M}^{++}$. Fixing $\infty \in \mathbb{M}^{00}$ (the point at infinity) one defines the Minkowski space $M_{\infty}$ as the set of points $x \in \mathbb{M}^{00}$ which are transversal to $\infty$. Fixing additionally $0 \in M_{\infty}$ (the origin of Lorentz system of coordinates) one can define the Poincare group $P_{\infty}$ extended by dilatations as the stabilizer $S U(2,2)_{\infty}$ of infinity; the intersection of stabilizers $S U(2,2)_{\infty} \cap S U(2,2)_{0}$ as the Lorentz group $L_{0, \infty}$ extended by the dilatation group $D_{0, \infty} ; L_{0, \infty}$ and $D_{0, \infty}$ as the commutator and centralizer of $S U(2,2)_{\infty} \cap S U(2,2)_{0}$ respectively. The group of Minkowski space translations $T_{\infty}$ is defined as a set of $\exp \chi$, where $\chi \in \mathscr{S} \mathscr{U}(2,2)$ is such that $\operatorname{Im} \chi$ $\subset \infty \subset \operatorname{Ker} \chi$. The group of four-acceleration $A_{0}$ is formed by $\exp \chi$, where $\operatorname{Im} \chi \subset 0$ $\subset \operatorname{Ker} \chi$. One also has the decomposition

$$
\mathscr{S} \mathscr{U}(2,2) \stackrel{C-K}{\cong} \mathscr{S} \mathscr{U}(2,2)^{*}=\mathscr{T}_{\infty}^{*} \oplus \mathscr{L}_{0, \infty}^{*} \oplus \mathscr{D}_{0, \infty}^{*} \oplus \mathscr{A}_{0}^{*},
$$

where $\mathscr{T}_{\infty}^{*} \cong \mathscr{A}_{0}, \mathscr{L}_{0, \infty}^{*}=\mathscr{L}_{0, \infty}, \mathscr{D}_{0, \infty}^{*} \cong \mathscr{D}_{0, \infty}$, and $\mathscr{A}_{0}^{*} \cong \mathscr{T}_{\infty}$ are dual spaces to corresponding Lie subalgebras (isomorphisms are given by the Cartan-Killing 
form). Everywhere below we shall use the representation: $\eta=i\left(\begin{array}{cc}0 & E \\ -E & 0\end{array}\right)$, $\infty=\left[\left(\begin{array}{l}\zeta \\ 0\end{array}\right): \zeta \in \mathbb{C}^{2}\right] \in \mathbb{M}^{00}$ and $0=\left[\left(\begin{array}{l}0 \\ \zeta\end{array}\right): \zeta \in \mathbb{C}^{2}\right]$. Then

$$
\mathbb{M}_{\infty}=\left\{z=\left[\left(\begin{array}{c}
Z \zeta \\
\zeta
\end{array}\right): \zeta \in \mathbb{C}^{2}\right]: Z \in \operatorname{Mat}_{2 \times 2}(\mathbb{C})\right\}
$$

and $z \in M_{\infty}$ iff $Z^{+}=Z, z \in \mathbb{M}^{++}$iff $\operatorname{det} \frac{1}{2 i}\left(Z-Z^{+}\right)>0$ and $\operatorname{Tr} \frac{1}{2 i}\left(Z-Z^{+}\right)>0$. The Lie subalgebras appearing in (4.1) will have then the form

$$
\begin{aligned}
\mathscr{T}_{\infty}^{*} & =\left\{\left(\begin{array}{cc}
0 & 0 \\
P & 0
\end{array}\right): P=P^{+} \in \operatorname{Mat}_{2 \times 2}(\mathbb{C})\right\}, \\
\mathscr{L}_{0, \infty}^{*} & =\left\{\left(\begin{array}{cc}
M & 0 \\
0 & -M^{+}
\end{array}\right): M \in \operatorname{Mat}_{2 \times 2}(\mathbb{C}), \operatorname{Tr} M=0\right\}, \\
\mathscr{D}_{0, \infty}^{*} & =\left\{\frac{1}{2} d\left(\begin{array}{cc}
E & 0 \\
0 & -E
\end{array}\right): d \in \mathbb{R}\right\}, \\
\mathscr{A}_{0}^{*} & =\left\{\left(\begin{array}{ll}
0 & A \\
0 & 0
\end{array}\right): A=A^{+} \in \operatorname{Mat}_{2 \times 2}(\mathbb{C})\right\} .
\end{aligned}
$$

The connection between Lorentz and matrix coordinates is given by Pauli matrices $\sigma_{\mu}$, where $\sigma_{0}=E=\left(\begin{array}{ll}1 & 0 \\ 0 & 1\end{array}\right)$ and $\mu=0,1,2,3$, i.e. $Z=z^{\mu} \sigma_{\mu}=\left(x^{\mu}+i y^{\mu}\right) \sigma_{\mu}$, $P=p^{\gamma} \sigma_{\gamma}, M=\frac{1}{2}\left(m^{0 k} \sigma_{k}-\varepsilon_{k i j} m^{k j} \sigma_{i}\right), A=a^{\gamma} \sigma_{\gamma}$, where $m^{k j}=-m^{j k}$ and $m^{0 k}=-m^{k 0}$.

The exhaustive discussion of phase space $\mathbb{M}^{++}$on the classical mechanical level is to be found in [11]. Here, let us only mention that the symplectic form is given by

$$
\omega_{\lambda}=i \lambda \frac{\partial^{2} \log y^{2}}{\partial z^{\gamma} \partial \bar{z}^{v}} d z^{\gamma} \wedge d \bar{z}^{v}, \quad 0 \neq \lambda \in \mathbb{R}
$$

and the momentum map $J_{\lambda}$ is of the form

$$
J_{\lambda}(z)=i \lambda\left[\begin{array}{cc}
\left(Z+Z^{+}\right)\left(Z-Z^{+}\right)^{-1}, & -2 Z\left(Z-Z^{+}\right)^{-1} Z^{+} \\
2\left(Z-Z^{+}\right)^{-1}, & -E-2\left(Z-Z^{+}\right)^{-1} Z^{+}
\end{array}\right] \in \mathscr{S} \mathscr{U}(2,2),
$$

where $z \in \mathbb{M}^{++}$. After decomposing $J_{\lambda}(z)$ according to (4.1) and passing to Lorentz coordinates, one obtains the four-momentum, angular momentum, dilatation and four-acceleration

$$
\begin{aligned}
p^{\mu} & =\lambda \frac{y^{\mu}}{y^{2}}=2 i \lambda \frac{z^{\mu}-\bar{z}^{\mu}}{(z-\bar{z})^{2}} \\
m^{\mu \nu} & =x^{\mu} p^{\nu}-x^{\nu} p^{\mu}=i \lambda \frac{1}{(z-\bar{z})^{2}}\left[\left(z^{\mu}+\bar{z}^{\mu}\right)\left(z^{\nu}-\bar{z}^{\nu}\right)-\left(z^{\nu}+\bar{z}^{v}\right)\left(z^{\mu}-\bar{z}^{\mu}\right)\right] \\
d & =2 x^{\mu} p_{\mu}=2 i \lambda \frac{\left(z^{\mu}+\bar{z}^{\mu}\right)\left(z_{\mu}-\bar{z}_{\mu}\right)}{(z-\bar{z})^{2}} \\
a^{v} & =-2 x \cdot p x^{v}+\left(x^{2}-\frac{\lambda^{2}}{p^{2}}\right) p^{\nu}=2 i \lambda \frac{\bar{z}^{2} z^{v}-z^{2} \bar{z}^{v}}{(z-\bar{z})^{2}}
\end{aligned}
$$


respectively. From (4.5) one has $y^{\mu}=\lambda \frac{p^{\mu}}{p^{2}}$ and $\omega_{\lambda}=d x^{\mu} \wedge d p_{\mu}$, and then $\left(x^{\mu}, p_{v}\right)$ becomes the canonical system of coordinates on $\mathbb{M}^{++}$.

In order to find the quantum mechanical description of the scalar massive conformal particle let us assume the tautological bundle $\mathbb{E}^{j}:=\otimes^{j} \wedge^{2} \mathbb{S} \rightarrow \mathbb{M}^{++}$as a quantum bundle $\mathbb{E}$, where $\mathbb{S}_{z}:=z$. The restriction of twistor form $\eta$ to $\mathbb{S}_{z}$ defines the metric structure on $\mathbb{S}$, thus $\mathbb{E}^{j}$ is a Hermitian line bundle. For the explicit calculations let us fix in $\mathbb{S}$ the holomorphic frame

$$
\left\{e_{1}(z):=\left[\begin{array}{c}
Z\left(\begin{array}{l}
1 \\
0
\end{array}\right) \\
\left(\begin{array}{l}
1 \\
0
\end{array}\right)
\end{array}\right], e_{2}(z):=\left[\begin{array}{c}
Z\left(\begin{array}{l}
0 \\
1
\end{array}\right) \\
\left(\begin{array}{l}
0 \\
1
\end{array}\right)
\end{array}\right]\right\}
$$

and define the holomorphic frame $s_{0}:=\stackrel{j}{\otimes} e_{1} \wedge e_{2}: \mathbb{M}^{++} \rightarrow \mathbb{E}^{j}$ in $\mathbb{E}^{j}$. The Hermitian metric on $\mathbb{E}^{j}$, expressed in $s_{0}$ is given by

$$
\varrho_{j, 0}=H\left(s_{0}, s_{0}\right)=2^{j} \operatorname{det}\left[\frac{1}{2 i}\left(Z-Z^{+}\right)\right]^{j} .
$$

Thus the scalar product (2.1) in the Hilbert space of quantum states $\mathscr{M}_{j, 0}$ attains the form

$$
\langle s, t\rangle_{j, 0}=2^{j+4} c \int_{\mathbb{M}^{++}} \overline{\psi(z)} \varphi(z) \operatorname{det}\left[\frac{1}{2 i}\left(Z-Z^{+}\right)\right]^{j} d^{4} x d^{4} y,
$$

where $s=\psi s_{0} \otimes d z^{0} \wedge \ldots \wedge d z^{3}, t=\varphi s_{0} \otimes d z^{0} \wedge \ldots \wedge d z^{3}$, and $c>0$ is a constant introduced for technical reasons. The condition of ampleness (2.2) for $\mathscr{M}_{j, 0}$ is satisfied when $j>-3$ (see [7]): therefore, in this case we can use the formalism developed in two the previous sections.

First of all let us observe that if one puts $\frac{\lambda}{h}=j$, then the symplectic form $\omega_{\lambda}$ is equal to the curvature form of the metric connection of the bundle $\mathbb{E}^{j}$. Because the parameter $\lambda$ has the dimension of the action we introduce the elementary unit $h$, which will be interpreted as the Planck constant. The momentum map components (4.5) are quantizable quantities, i.e. belong to $C_{F F}$. Hence, from (2.9) one finds

$$
\begin{aligned}
p_{\mu} & =-i h \frac{\partial}{\partial z^{\mu}} \\
\hat{m}_{\mu \nu} & =-i h\left(z_{\mu} \frac{\partial}{\partial z^{v}}-z_{v} \frac{\partial}{\partial z^{\mu}}\right), \\
\hat{d} & =-i h 2 z^{\mu} \frac{\partial}{\partial z^{\mu}}-2(j+4) h i \\
\hat{a}_{v} & =-i h\left(z^{2} \delta_{v}^{\beta}-2 z_{v} z^{\beta}\right) \frac{\partial}{\partial z^{\beta}}+2 i h(j+4) z_{v} .
\end{aligned}
$$


The conformal group $S U(2,2)$ acts on $\mathbb{E}^{j} \rightarrow \mathbb{M}^{++}$, preserving its holomorphic and metric structure. This enables us to define the unitary representation $T_{j}: S U(2,2)$ $\rightarrow U\left(\mathscr{M}_{j, 0}\right)$ of the conformal group:

$$
\left(T_{j}(g) \psi\right)(z):=\operatorname{det}(C Z+D)^{-j-4} \psi\left[(A Z+B)(C Z+D)^{-1}\right],
$$

where $g^{-1}=\left(\begin{array}{ll}A & B \\ C & D\end{array}\right) \in S U(2,2)$ and $A, B, C, D \in \operatorname{Mat}_{2 \times 2}(\mathbb{C})$ (see [7]). It is easy to check that (4.7) taken without the factor $-i$ gives the generators of $T_{j}$. Thus, by Stone's theorem (see [21]), $\hat{p}_{\mu}, \hat{m}_{\mu \nu}, \hat{d}$, and $\hat{a}_{v}$ are selfadjoint operators.

The reproducing kernel function for $\mathscr{M}_{j, 0}$ is calculated in [7]. For $c=2^{-3(j+4)}$ it has the form

$$
K_{j, 0}(\bar{w}, z)=\left\{\left[\frac{1}{2 i}(z-\bar{w})\right]^{2}\right\}^{-j-4}=\left[c_{j}^{2} \frac{\pi}{2} \Gamma(j+3) \Gamma(j+4)\right]^{-1} \int_{C_{+}} \overline{\psi_{k}(w)} \psi_{k}(z) d^{4} k,
$$

where $C_{+}$denotes the future cone and $\psi_{k}(z):=c_{j}\left(k^{2}\right)^{\frac{j}{2}+1} e^{i k z}$ is the generalized eigenvector of the four-momentum operator, $\hat{p}_{\gamma} \psi_{k}(z)=h k_{\gamma} \psi_{k}(z)$. In order to have $\left\langle\psi_{k}, \psi_{k^{\prime}}\right\rangle_{j, 0}=\delta_{4}\left(k-k^{\prime}\right)$, we put

$$
c_{j}=2^{j}\left[\left(\frac{\pi}{2}\right)^{5} \Gamma(j+1) \Gamma(j+2)\right]^{-1 / 2} .
$$

The Laplace transform

$$
\psi(z)=\int_{C_{+}} \varphi(k) \psi_{k}(z) d^{4} k,
$$

where $\varphi \in L^{2}\left(C_{+}, d^{4} k\right)$ plays here a similar role as the Fourier transform in Schrödinger quantum mechanics, i.e. it allows us to pass from momentum to holomorphic representation. Using the momentum representation it is easy to compute the average values of quantities in coherent states $K_{j, 0}(\bar{z}, \cdot)$. In this way one obtains

$$
\frac{\left\langle K_{j, 0}(\bar{z}, \cdot), \hat{f} K_{j, 0}(\bar{z}, \cdot)\right\rangle_{j, 0}}{\left\langle K_{j, 0}(\bar{z}, \cdot), K_{j, 0}(\bar{z}, \cdot)\right\rangle_{j, 0}}=\left(1+\frac{4}{j}\right) f(z)
$$

where $f$ is an element of the conformal Poisson algebra, i.e. $f$ is a linear combination of generators (4.5).

As one would expect, the weight function $\varrho_{j, 0}$ fulfills the condition (2.22) for a suitably chosen constant $C$. Therefore,

$$
a_{j, 0}(\bar{w}, z)=\left\{\frac{\left[(z-\bar{z})^{2}(w-\bar{w})^{2}\right]^{1 / 2}}{(z-\bar{w})^{2}}\right\}^{j+4}
$$

is the probability amplitude that the scalar massive conformal particle after detection in the coherent state $z$ will be detected in the other coherent state $w$. According to (4.8) the $\psi_{k}(z)$, where $k \in C_{+}$, form a complete system of generalized states. Hence, one can interpret

$$
a_{j, 0}(z, k):=\left[\frac{\pi}{2} \Gamma(j+3) \Gamma(j+4)\right]^{-1 / 2}\left(k^{2}\right)^{\frac{j}{2}+1}\left[\left(\frac{z-\bar{z}}{2 i}\right)^{2}\right]^{\frac{j+4}{2}} e^{i k z}
$$


as the transition probability amplitude from the state with a given fourmomentum $h k$ to the coherent state $z$. Using (4.12) one can rewrite (4.8) in the physically more transparent form

$$
a_{j, 0}(\bar{w}, z)=\int_{C_{+}} a_{j, 0}(z, k) \overline{a_{j, 0}(w, k)} d^{4} k .
$$

Consequently, the transition probability amplitude for a scalar massive relativistic particle, i.e. a particle which in all processes satisfy $(h k)^{2}=p^{2}=(m c)^{2}=$ const is given by

$$
\begin{aligned}
a_{j, 0, m}(\bar{w}, z):= & \int_{C_{+}} a_{j, 0}(\bar{z}, k) \overline{a_{j, 0}(w, k)} \delta\left(k^{2}-\left(\frac{m c}{h}\right)^{2}\right) d^{4} k=\left[\frac{\pi}{2} \Gamma(j+3)\right. \\
& \times \Gamma(j+4)]^{-1} j^{2(j+4)}\left(\frac{m c}{h}\right)^{-4} \int_{C_{+}} e^{i k(z-\bar{w})} \delta\left(k^{2}-\left(\frac{m c}{h}\right)^{2}\right) d^{4} k
\end{aligned}
$$

It is remarkable that the condition of mass constancy $p(\tau)^{2}=(m c)^{2}=$ const defining the relativistic process

$$
\left[\tau_{i}, \tau_{f}\right] \ni \tau \stackrel{\gamma}{\longrightarrow} K_{j, 0}(\bar{z}(\tau), \cdot) \in \mathscr{M}_{j, 0}
$$

is equivalent to the unitarity condition $\left\|K_{j, 0}(\bar{z}(\tau), \cdot)\right\|=$ const. Specifying the formula (2.24) to the case considered here one finds that

$$
a_{j, 0}(\gamma ; \bar{w}, z)=\exp \left[-\frac{i}{h} \int_{\gamma} \pi_{\mu} d x^{\mu}\right]
$$

is the probability amplitude of transition of the scalar massive conformal particle along the path $\gamma$ beginning at $z=\gamma\left(\tau_{i}\right)$ and ending at $w=\gamma\left(\tau_{f}\right)$, where the $\pi_{\mu}:=\left(1+\frac{4}{j}\right) p_{\mu}$ is the average four-momentum obtained from (4.10).

Let us now make some remarks about the coherent states $K_{j, 0}(\bar{z}, \cdot) \in \mathscr{M}_{j, 0}$, where $z \in \mathbb{M}^{++}$. The average values of all kinematic quantities $\hat{f}$ computed in $K_{j, 0}(\bar{z}, \cdot)$, see (4.10), are equal to their classical counterparts up to factor $1+\frac{4}{j}$. On the other hand it is a matter of simple calculation to show that the probability density $\left|a_{j, 0}(\bar{z}, w)\right|^{2}$ to detect the scalar massive conformal particle in the state $K_{j, 0}(\bar{z}, \cdot)$ in the point $w \in \mathbb{M}^{++}$is concentrated around the point $z$ and attains in it the maximal value. As a consequence of this one can consider the scalar massive conformal particle as the object localized at the point $z^{\mu}=x^{\mu}+i(j+4) \frac{h}{m c} \frac{\pi^{\mu}}{m c}$, where $(m c)^{2}=\pi^{2}$. If the Compton wavelength $\frac{h}{m c}$ and average four-velocity $\frac{\pi^{\mu}}{m c}$ are small, i.e. in the case of low energy, the scalar massive conformal particle is localized in a region of $\mathbb{M}^{++}$closed to Minkowski spacetime $\left(y^{\mu} \approx 0\right)$. It is interesting to mention that $\mathbb{M}^{00}$ is the Shilov boundary of $\mathbb{M}^{++}$(see [15]) and the maximal value of $K_{j, 0}(\bar{z}, \cdot)$ is attained at the spacetime point $x=\operatorname{Re} z \in M_{\infty}$. Let us also notice that because of

$$
\frac{T_{j}(g) K_{j, 0}(\bar{z}, \cdot)}{\left\|T_{j}(g) K_{j, 0}(\bar{z}, \cdot)\right\|}=\left[\frac{\operatorname{det}\left(A-Z^{+} C\right)}{\left|\operatorname{det}\left(A-Z^{+} C\right)\right|}\right]^{-j-4} \frac{K_{j, 0}(\overline{g z}, \cdot)}{K_{j, 0}(\overline{g z}, g z)^{1 / 2}},
$$


the imbedding $\mathscr{K}_{j, 0}: \mathbb{M}^{++} \rightarrow \mathbb{C P}\left(\mathscr{M}_{j, 0}\right)$ is the conformally equivariant map of classical phase space into quantum phase space.

We shall now discuss the interaction of the scalar massive conformal particle with the external field. In accordance with Sect. 3, the external field is described by the deformation (3.2) of the holomorphic and metric structure of the bundle $\mathbb{E}^{j}$. Applying the definition (3.6) to the present case we find the Lagrangian $d L$ which, expressed in the canonical coordinates $\left(x^{\mu}, \pi_{v}\right)$, is given by

$$
\begin{aligned}
d L= & -\pi_{\mu} d x^{\mu}+\frac{h}{2} \log \frac{\pi^{2}}{\left(m_{0} c\right)^{2}} d t+\frac{h}{2(j+4)} A(x, \pi) d t \\
& +\frac{h}{2} \frac{\partial A}{\partial y^{\mu}}(x, \pi) d x^{\mu}-\frac{h^{2}}{2}(j+4) \frac{\partial A}{\partial x^{\mu}}(x, \pi) d\left(\frac{\pi^{\mu}}{\pi^{2}}\right),
\end{aligned}
$$

where we put $f=\frac{1}{2(j+4)} \log$ and $k_{0}=\left[\frac{m_{0} c}{(j+4) h}\right]^{2(j+4)}$. Since the deformation of the reproducing kernel $A=A(x, \pi)$ results from the deformation $B=B(x, \pi)$ of the structure of the Hermitian vector bundle $\mathbb{E}^{j}[$ see (3.3)] we shall interpret the three last parts of (4.17) as the interaction Lagrangian $d L_{I}$. The remaining two parts will form the Lagrangian $d L_{0}$ for the free scalar massive conformal particle. From the variational principle $\delta \int d L_{0}=0$ one obtains $\frac{d x}{d t}=h \frac{\pi^{\mu}}{\pi^{2}}$ and $\frac{d \pi^{\mu}}{d t}=0$. Thus, it is reasonable to assume that $s:=\frac{h}{m c} t$ is the particle proper time measured in natural units given by the Compton wavelength.

In order to study the Lagrangian (4.17) in the low energy region $\left(y^{\mu} \approx 0\right)$ one needs the expansion.

$$
A(x, y)=A(x)+A_{\mu}(x) y^{\mu}+A_{\mu \nu}(x) y^{\mu} y^{v}+\ldots
$$

Substituting (4.18) into (4.17) one finds

$$
d L=-\pi_{\mu} d x^{\mu}+\frac{h}{2} \log \frac{\pi^{2}}{\left(m_{0} c\right)^{2}} d t+\frac{h}{2(j+4)} A(x) d t+\frac{h}{2} A_{\mu}(x) d x^{\mu}+\mathcal{O}\left(h^{2}\right),
$$

where $\mathcal{O}\left(h^{2}\right) d t$ is the part of the Lagrangian which contains the second and further orders of the Planck constant. Neglecting $\mathcal{O}\left(h^{2}\right) d t$ and assuming that the average relativistic mass $m$ of the particle does not change during the interaction and that $m=m_{0}$ (such an assumption seems to be acceptable if the low energy case is considered), we obtain the Lagrangian for the charged scalar massive particle in the external electromagnetic field of the four-potential $-\frac{h c}{2 e} A_{\mu}(x)$, where $e$ is the electric charge of the particle. The term $-\frac{h}{2(j+4)} A(x)$ can be interpreted as a scalar potential for some additional (nonelectromagnetic) force acting on the particle. According to the assumption $\pi^{2}=\left(m_{0} c\right)^{2}$, the second term in $d L$ disappears. Hence we see that the interaction with the electromagnetic field is a linear approximation - in the sense of expansion (4.18) - of the interaction with the holomorphic field. The effects that would expose the difference between the 
holomorphic theory and Maxwell electrodynamics depend on the higher order terms of the expansion (4.18), and because of that they can be noticeably large only in the high energy region. Therefore, for the low energy case it is reasonable to seek an approximate calculus that would relate the low order terms of the expansion

$$
B(x, y)=B(x)+B_{\mu}(x) y^{\mu}+B_{\mu \nu}(x) y^{\mu} y^{\nu}+\ldots
$$

and such of (4.18). The expansion of the field equation (3.3) with respect to $y^{\mu}$ gives the infinite sequence

$$
\begin{gathered}
B(x)+A(x)=0, \\
B_{\mu}(x)+A_{\mu}(x)=0, \\
B_{\mu \nu}(x)+A_{\mu \nu}(x)=j\left[\eta_{\mu \nu}\left(\frac{1}{2} \square_{x} B(x)+B_{\alpha}^{\alpha}(x)\right)-\frac{\partial^{2} B(x)}{\partial x^{\mu} \partial x^{\nu}}-B_{\mu \nu}(x)\right],
\end{gathered}
$$

of equations on the fields $B(x), B_{\mu}(x), B_{\mu \nu}(x), \ldots$. The above interpretation of $A_{\mu}(x)$ raises a fundamental question. Namely, is there any relation between the first order approximation of (3.3) and Maxwell equations?

Finally let us calculate the probability amplitude $a_{j, B}\left(k_{f}, k_{i}\right)$ of the transition of the scalar massive conformal particle from the state $\psi_{k_{i}}$ to the state $\psi_{k_{f}}$ in the external field $B(x, \pi)$. In order to attain it, we shall consider the case when the field $B(x, \pi)$ is concentrated in the bounded region of phase space. In accordance with the above considerations, the transition probability amplitude is given by

$$
a_{j, B}\left(k_{f}, k_{i}\right)=\left\langle\psi_{k_{\imath}}, \psi_{k_{f}}\right\rangle_{j, B}=2^{-2(j+4)} \int_{\mathbb{M}^{++}} \overline{\psi_{k_{i}}}(z) \psi_{k_{f}}(z)\left(y^{2}\right)^{j} e^{B} d^{4} x d^{4} y .
$$

Substituting (4.20) into (4.22), one obtains

$$
\begin{aligned}
a_{j, B}\left(k_{f}, k_{i}\right)= & \delta_{4}\left(k_{f}-k_{i}\right)+2^{2 j+5}(j+2) \frac{\left(k_{i}^{2} k_{f}^{2}\right)^{\frac{j}{2}+1}}{\left[\left(k_{i}+k_{f}\right)^{2}\right]^{j+3}} \hat{B}_{\mu}\left(k_{f}-k_{i}\right)\left(k_{i}^{\mu}+k_{f}^{\mu}\right) \\
& -2^{2 j+4}(j+2) \frac{k_{i}^{2} k_{f}^{2}}{\left[\left(k_{i}+k_{f}\right)^{2}\right]^{j+3}}\left(\widehat{B_{\mu} B_{v}+2} B_{\mu v}\right)\left(k_{f}-k_{i}\right) \\
& \times\left[\eta^{\mu \nu}-\frac{2(j+3)\left(k_{i}^{\mu}+k_{f}^{\mu}\right)\left(k_{i}^{v}+k_{f}^{v}\right)}{\left(k_{i}+k_{f}\right)^{2}}\right]+\mathcal{O}\left(h^{3}\right),
\end{aligned}
$$

where $\widehat{B}_{\mu}, \widehat{B_{\mu} B_{v}+2} B_{\mu v}, \ldots$ are Fourier transforms of the corresponding quantities. $\mathcal{O}\left(h^{3}\right)$ denotes the terms in the Planck constant of the order higher than two and they are negligible when only a low energy process is considered. The $a_{j, B}\left(k_{f}, k_{i}\right)$ depends also on the index $j>-3$, which could be interpreted here as a regularization parameter. Considering the singular case $j=-3$ (for which $\left.\pi^{\mu}=h \frac{y^{\mu}}{y^{2}}\right)$ of (4.22), and postulating that the mass of the scalar massive conformal particle does not change as the result of interaction, one obtains up to the constant factor the standard formula for the transition probability amplitude, see (9.24) in [2] as the linear approximation of (4.23). 
Acknowledgements. I would like to thank W. Lisiecki, M. Sंwıęckı, L. S. Woronowıcz for useful discussions. Special mention must be made of Prof. K. Maurin for his deep interest in this work.

\section{References}

1. Atiyah, M.F., Drinfeld, V.G., Hitchin, N.J., Manin, Yu.I.: Construction of instantons. Phys. Lett. 65A, 185-187 (1978)

2. Bjorken, J.D., Drell, S.D.: Relativistic quantum mechanics. New York: McGraw-Hill (1964)

3. Gaweedzki, K.: Fourier-like kernels in geometric quantization. CXXV Dissertationes Mathematicae, Warszawa (1976)

4. Griffiths, P., Harris, J.: Principles of algebraic geometry. New York: Wiley 1978

5. Hua Lo-Keng: Harmonic analysis of functions of several complex variables in the classical domains. Peking: Science Press 1958. Transl. Math. Monlgraph 6, Providence, R.I.: Am. Math. Soc. 1963

6. Hurt, N.E.: Geometric quantization in action. Dordrecht: Reidel 1983

7. Jakobsen, H.P., Vergne, M.: Wave and Dirac operators, and representations of the conformal group. J. Funct. Anal. 24, 52-106 (1977)

8. Kobayashi, S.: Geometry of bounded domains. Trans. Am. Math. Soc. 92, 267-290 (1959)

9. Kostant, B.: Quantization and unitary representation. Lecture Notes in Mathematics, Vol. 170. pp. 87-208. Berlin, Heidelberg, New York: Springer 1970

10. Lisiecki, W., Odzijewicz, A.: Twistor flag spaces as phase spaces of conformal particles. Lett. Math. Phys. 3, 325-334 (1979)

11. Odzijewicz, A.: A conformal holomorphic field theory. Commun. Math. Phys. 107, 561-575 (1986)

12. Pasternak, Z.: On dependence of the reproducing kernel on the weight of integration (preprint)

13. Penrose, R.: The twistor programme. Rep. Math. Phys. 12, 65-76 (1977)

14. Penrose, R., MacCallum, M.A.H.: Twistor theory: an approach to the quantization of fields and space-time. Phys. Rep. 6, No. 4, 241-316 (1972)

15. Rühl, W.: Distributions on Minkowski space and their connection with analytic representations of the conformal group. Commun. Math. Phys. 27, 53-86 (1972)

16. Simms, D.J.: Geometric quantization of energy levels in the Kepler problem. Conv. di Geom. Simp. e Fis. Mat. INDAM, Rome (1973)

17. Skwarczyński, M.: Biholomorphic invariants related to the Bergman Functions. Dissertationes Math. 173, PWN, Warszawa, 1-64 (1980)

18. Souriau, J.M.: Structure des Systemes dynamiques. Paris: Dunod 1970

19. Shabat, B.V.: Vvedenie v kompleksnyj analiz. Moskva: Nauka 1969

20. Yau, S.-T.: On the Ricci curvature of a compact Kähler manifold and the complex MongeAmpère equation. I. Commun. Pure Appl. Math. XXXI, 339-411 (1978)

21. Yosida, K.: Functional analysis. Berlin, Heidelberg, New York: Springer 1965

Communicated by R. Haag

Received December 5, 1986; in revised form July 16, 1987 
